[Article]

www.whxb.pku.edu.cn

\title{
$\mathrm{CdS}$ 纳米棒的制备、表征及其形成机理
}

\author{
陈书堂张小玲* 侯晓森 周 琦 \\ (北京理工大学理学院化学系, 北京 100081)
}

\begin{abstract}
摘要：以三辛基膦(TOP)为单一配位溶剂, 二水合乙酸镉和硫粉为前驱体, 用高温热解的方法制备 $\mathrm{CdS}$ 纳米棒. 通过 X 射线衍射(XRD)、透射电镜(TEM)、高分辨透射电镜(HRTEM)、紫外-可见(UV-Vis)分光光度计、菼光(PL) 光谱、傅里叶变换红外(FTIR)光谱和核磁共振磷谱 $\left({ }^{31} \mathrm{P}\right.$ NMR)等方法对样品的结构、形貌和光学特性进行了表征. 考察了前驱体 $\mathrm{Cd} / \mathrm{S}$ 摩尔比和反应物浓度对硫化镉纳米结构的影响. 实验结果表明, 该法制备的 $\mathrm{CdS}$ 纳米棒为 纤锌矿结构, 直径为 $4.0 \mathrm{~nm}$, 长度为 $28.0 \mathrm{~nm}$, 沿[001]方向择优生长, 具有量子限域效应. 同时, 对 $\mathrm{CdS}$ 纳米晶的 形成机理进行了初步的探讨.
\end{abstract}

关键词：纳米棒; 硫化镉; 三辛基膦; 合成; 菼光 中图分类号：O649

\section{Synthesis, Characterization and Formation Mechanism of CdS Nanorods}

\author{
CHEN Shu-Tang ZHANG Xiao-Ling* HOU Xiao-Miao ZHOU Qi \\ (Department of Chemistry, School of Science, Beijing Institute of Technology, Beijing $\quad$ 100081, P. R. China)
}

\begin{abstract}
CdS nanorods were successfully synthesized by pyrolysis using trioctylphosphine (TOP) as a coordinating solvent. For this pyrolysis, cadmium acetate dihydrate and sulfur powder were used as the cadmium source and sulfur source, respectively. The structure, morphology, and optical properties of the prepared samples were characterized by $\mathrm{X}$-ray diffraction (XRD), transmission electron microscopy (TEM), high-resolution transmission electron microscopy (HRTEM), ultraviolet-visible (UV-Vis) spectroscopy, photoluminescence (PL), Fourier transform infrared (FTIR) spectroscopy, and nuclear magnetic resonance (NMR) ${ }^{31} \mathrm{P}$ spectroscopy. The effects of different molar ratios of Cd-to-S and the reaction concentrations on the morphology and size of the CdS nanostructures were investigated. The results indicated that the wurtzite CdS nanorods, with a typical diameter of $4.5 \mathrm{~nm}$ and a length of up to $28.0 \mathrm{~nm}$, had a preferential [001] growth direction and displayed a quantum confinement effect. We also proposed a possible formation mechanism for the CdS nanocrystals based on the experimental results.
\end{abstract}

Key Words: Nanorods; Cadmium sulfide; Trioctylphosphine; Synthesis; Photoluminescence

一维半导体纳米晶不同于块材的奇特电学和 光学性质, 在发光二极管 ${ }^{[1-4]}$ 、高效太阳能电池 ${ }^{[5-8]} 、$ 量 子点微型激光器 ${ }^{[9-11]}$ 和基于光致发光的多色生物标 签 ${ }^{[12-14]}$ 等领域有巨大的应用前景, 因此, 合成一维半 导体纳米晶已成为近年来人们研究的热点.

一维半导体纳米晶的制备方法可分为物理法和
化学法.一般的物理方法需要精密昂贵的大型设备 和极端苛刻的实验条件. 相比而言, 化学法则具有易 于操作、价格低廉和简单有效的特点. 目前制备一维 半导体纳米晶的液相化学方法主要有: 溶剂热法 ${ }^{[15]}$ 、

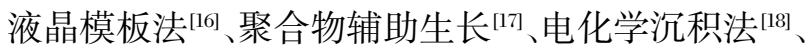
气相沉积法 ${ }^{[19]}$ 、部分阳离子交换的方法 ${ }^{[20]}$ 和胶体化

Received: July 17, 2009; Revised: November 23, 2009; Published on Web: December 22, 2009.

*Corresponding author. Email: zhangxl@bit.edu.cn; Tel: +86-10-88875298.

The project was supported by the National Natural Science Foundation of China (0975012) and Program of Introducing Talents of Discipline to Universities, China (B07012)

国家自然科学基金(20975012)和高等学校学科创新引智计划(B07012)资助项目

C Editorial office of Acta Physico-Chimica Sinica 
学法 ${ }^{[21-27]}$ 等. 自从 1993 年 Murray 等 ${ }^{[21]}$ 报道胶体化学 法制备半导体纳米晶以来, 该法成为目前应用最广 泛的方法. 例如, Peng 等 ${ }^{[23]}{ }^{\left[耳^{2}\right.}$ 早利用已基亚磷酸与镉 原子较强的配位能力来辅助合成 $\mathrm{CdSe}$ 纳米棒; Cheon 等 ${ }^{[24}$ 利用十八胺作为配体高温分解单分子前 驱体合成了直径大于 $6 \mathrm{~nm}$ 的 $\mathrm{CdS}$ 纳米棒; Prashan 等 ${ }^{[2]}$ 利用配体对 $\mathrm{CdSe}$ 团簇的配位能力不同而合成 $\mathrm{CdSe}$ 纳米线.

为使一维半导体纳米晶的制备和形成机理研究 起来更为方便, 人们力图在合成过程中使用尽可能 少的配位溶剂. 本文以三辛基膦为单一配位溶剂, 醋 酸镉和硫粉作为前驱体, 成功合成了 $\mathrm{CdS}$ 纳米棒. 该制备工艺不仅简单高效, 而且容易推测 $\mathrm{CdS}$ 纳米 棒的形成和生长机理.

\section{1 实验部分}

\section{1 试剂、仪器及测试条件}

三辛基膦(TOP, 90\%)购自 Alfa Aesar 公司, 二水 合醋酸镉(分析纯, 98\%), 硫粉(99.5\%), 甲苯(99.8\%), 无水甲醇(99.9\%)和无水乙醇( $99.7 \%)$ 均购自北京化 学试剂公司, 所有试剂未经进一步纯化. 纳米晶结构 通过 X 射线衍射仪(D/MAX 2400, 日本 Rigaku 公司) 进行分析; 纳米晶的形貌与尺寸大小用透射电子显 微镜(FET TECNAI F30, 荷兰 FEI 公司)进行表征与 分析; 并采用紫外-可见分光光度计(PE Lambda 35, 美国 PE 公司), 苂光光度计 (F-4500, 日本日立公司) 和傅里叶变换红外光谱仪(Bruker Equiinox 55, 德国 Bruker 公司)在室温下测试其光学性质. 反应物的磷 谱用核磁共振光谱仪 (Bruker A VANCEII, 德国 Bruker 公司)在频率为 $400 \mathrm{MHz}$ 下分析.

\section{$1.2 \mathrm{CdS}$ 纳米棒的合成}

将 $0.15 \mathrm{mmol}(40.0 \mathrm{mg})$ 的醋酸镉与 $3 \mathrm{~mL}$ TOP加
人到 $25 \mathrm{~mL}$ 的三口颈瓶中, 在氮气保护和剧烈的磁 力搅拌下加热至 $260{ }^{\circ} \mathrm{C}$, 恒温 $5 \mathrm{~min}$, 用注射器慢速 $\left(0.5 \mathrm{~mL} \cdot \mathrm{min}^{-1}\right)$ 加人硫溶液 $(24.0 \mathrm{mg}$ 硫粉溶解在 1 $\mathrm{mL}$ TOP 中). 在此温度下反应 $5 \mathrm{~min}$ 后, 溶液颜色由 无色透明变为浅黄色, 然后逐渐变为橙黄色, 分别在 不同反应时间间隔取样进行动力学测试. 反应 $4 \mathrm{~h}$ 后停止加热, 待溶液冷却至室温后, 加人一定体积的 无水甲醇, 使 $\mathrm{CdS}$ 纳米晶沉降, 在 $6000 \mathrm{r} \cdot \mathrm{min}^{-1}$ 下离 心, 用无水乙醇洗涤 $\mathrm{CdS}$ 纳米晶 5 次后, 将 $\mathrm{CdS}$ 纳 米晶分散在甲苯中, 密闭干燥保存.

\section{2 结果与讨论}

\section{$2.1 \mathrm{CdS}$ 纳米棒的结构和形貌}

实验考察了 $\mathrm{Cd} / \mathrm{S}$ 配比对所制备 $\mathrm{CdS}$ 纳米晶形 貌和结构的影响. 在反应过程中, 保持其它条件不 变, 只改变镉的量, 观察所形成纳米晶的形貌和结 构. 图 1 为不同 $\mathrm{Cd} / \mathrm{S}$ 摩尔比下制备的 $\mathrm{CdS}$ 纳米晶 透射电镜图. 从图 1 中可以看出, $\mathrm{Cd} / \mathrm{S}$ 摩尔比为 $2: 1$ 和 1:1 时, 容易得到枝状的 $\mathrm{CdS}$ 纳米晶; 当 $\mathrm{Cd} / \mathrm{S}$ 配 比减小到 1:5 时, 可以得到单分散的棒状 $\mathrm{CdS}$ 纳米 晶. 我们认为, 在反应初期, 随着 $\mathrm{Cd} / \mathrm{S}$ 摩尔比的减 小, 由于 $\mathrm{CdS}$ 生长晶核数较少, 每个 $\mathrm{CdS}$ 生长晶核 平均可接受的 $\mathrm{CdS}$ 生长基元较多, 这与合成 $\mathrm{CdSe}$ 纳米棒的报道相似 ${ }^{[26]}$. 因此, $\mathrm{Cd} / \mathrm{S}$ 摩尔比对 $\mathrm{CdS}$ 纳 米晶的形貌有很大影响.

为了制备更大长径比的 $\mathrm{CdS}$ 纳米棒, 将 $\mathrm{Cd}$ 和 $\mathrm{S}$ 前驱体的量各减少一倍 $(20 \mathrm{mg}$ 醋酸镉和 $12 \mathrm{mg}$ 硫 粉), 反应 $4 \mathrm{~h}$ 后得到直径为 $4.5 \mathrm{~nm}$, 长度为 $28.0 \mathrm{~nm}$ 的 CdS 纳米棒(图 2a). 从对应的高分辨透射电镜图 (图 2b)中可以看到清晰的 CdS 晶格条纹, 垂直于生 长方向的晶格条纹间距为 $0.335 \mathrm{~nm}$, 这与纤锌矿 $\mathrm{CdS}$ 的(002) 晶面间距一致 ${ }^{[2]]}$, 证明 $\mathrm{CdS}$ 纳米棒沿
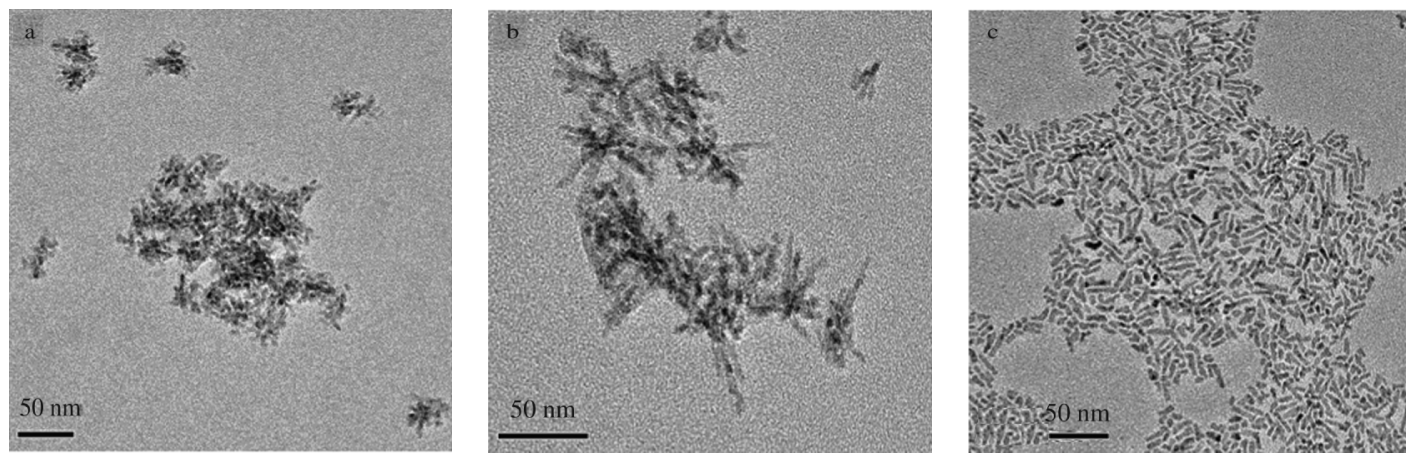

图 1 加入不同 $\mathrm{Cd} / \mathrm{S}$ 摩尔比得到的 CdS 样品的 TEM 照片

Fig.1 TEM images of the as-prepared CdS sample with different molar ratios of Cd-to-S 

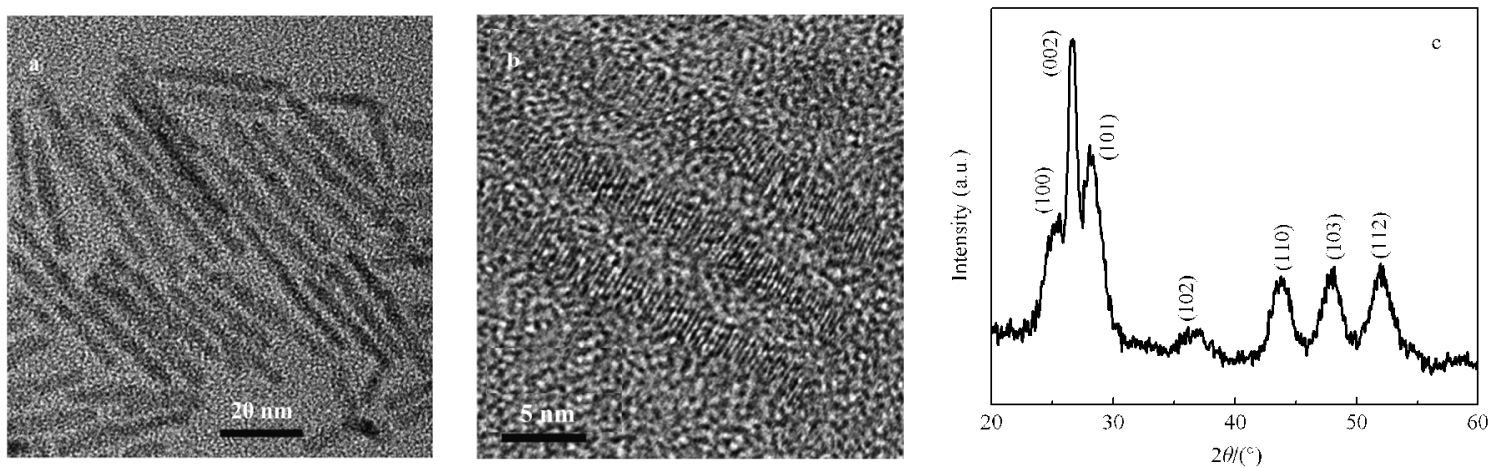

图 $2 \mathrm{CdS}$ 纳米棒的 TEM 照片(a), 相应的 HRTEM 照片(b)和 X 射线衍射图(c)

Fig.2 TEM image of the as-prepared CdS nanorods (a), corresponding HRTEM image (b) and XRD pattern (c) preparation condition: $20 \mathrm{mg}$ cadmium acetate in $3 \mathrm{~mL}$ trioctylphosphine (TOP) and $12 \mathrm{mg}$ S powder in $1 \mathrm{~mL}$ TOP reacting at $260{ }^{\circ} \mathrm{C}$ for $4 \mathrm{~h}$

[001]方向生长. 图 2c 为该样品对应的 XRD 谱图. 谱 图中出现的各晶面衍射峰的位置均与 CdS 六方纤 锌矿构型的 JCPDS 标准卡片(41-1049)的衍射峰位 置相符. (002)晶面对应的衍射峰比较尖锐, 进一步 证明了 $\mathrm{CdS}$ 纳米棒沿 [001]方向择优生长.

\section{$2.2 \mathrm{CdS}$ 纳米棒的光学特性}

实验考察了所制备 $\mathrm{CdS}$ 纳米棒的吸收光谱和 苂光光谱. 图 3a 为 $\mathrm{CdS}$ 纳米棒分散于甲苯中的紫 外-可见吸收光谱, 由图 3a 可见, $\mathrm{CdS}$ 纳米棒在 447 $\mathrm{nm}$ 处有明显的吸收峰, 相对于 CdS 块体材料 515 $\mathrm{nm}$ 的特征吸收峰有 $68 \mathrm{~nm}$ 的蓝移, 这是由于纳米 棒的量子限域效应引起的 ${ }^{[27]}$. 图 $3 \mathrm{~b}$ 给出了 $\mathrm{CdS}$ 纳米 棒样品的荧光发射谱图. 谱图中有两个发射峰, 其中 位于 $466 \mathrm{~nm}$ 处的发射峰属于带隙发射, 是由于电 子-空穴对复合引起的; 位于 $606 \mathrm{~nm}$ 处的发射峰属 于表面态发射, 是由表面结构缺陷引起的 ${ }^{229}$. 图 3c为 红外光谱, 通过分析表明, CdS 纳米棒的表面吸附了 三辛基膦分子.

\section{$2.3 \mathrm{CdS}$ 纳米晶的形成机理探讨}

硫粉和三辛基膦在室温下超声或者搅拌即可
生成三辛基硫化膦(TOPS), 其 ${ }^{31} \mathrm{P}$ 核磁共振谱图在 48.5303 出现一单峰(见 Supporting Information, 附图 1 , 可从物理化学学报网站 http://www.whxb.pku.edu. $\mathrm{cn}$ 免费下载), 表明制备的三辛基硫化膦比较纯, 无 其它三辛基膦的衍生物生成. 将三辛基硫化膦在 $260{ }^{\circ} \mathrm{C}$ 下加人到镉的前驱体中, $\mathrm{CH}_{3} \mathrm{COO}-\mathrm{Cd}$ 作为镉 离子的路易斯酸易于与富电子的三辛基硫化膦配 位, 生成过渡态复合结构 $\mathrm{CH}_{3} \mathrm{COO}-\mathrm{Cd}-\mathrm{S}-\mathrm{P}\left(\mathrm{CH}_{3}\right)$ (见 反应(1)). $\mathrm{CH}_{3} \mathrm{COO}-\mathrm{Cd}$ 与三辛基硫化膦配位后减弱 了硫磷键 ${ }^{[0-31]}$, 会导致硫磷键的断裂, 生成过渡态复 合结构 $\mathrm{CH}_{3} \mathrm{COO}-\mathrm{Cd}-\mathrm{S}$, 如反应(2)所示. $260{ }^{\circ} \mathrm{C}$ 下反 应 $5 \mathrm{~min}$ 后, 溶液颜色由无色透明变为淡黄色, 表明 有 $\mathrm{CdS}$ 单体生成(见反应(3)). 在该温度下反应 $4 \mathrm{~h}$ 后 取样进行 ${ }^{31} \mathrm{P}$ 核磁共振测试发现, ${ }^{31} \mathrm{P}$ 核磁共振图谱 (见 Supporting Information, 附图 2)主要在-30.671、 $32.661 、 34.832 、 48.524 、 49.057$ 和 57.455 有峰出现, 其中-30.671 和 48.524 处的峰分别对应三辛基膦 和三辛基硫化膦. 对纯的三辛基氧化膦(TOPO)进行 ${ }^{31} \mathrm{P}$ 核磁共振测试发现在 49.112 处有一单峰(见 Supporting Information, 附图 3), 和附图 2 中 49.057
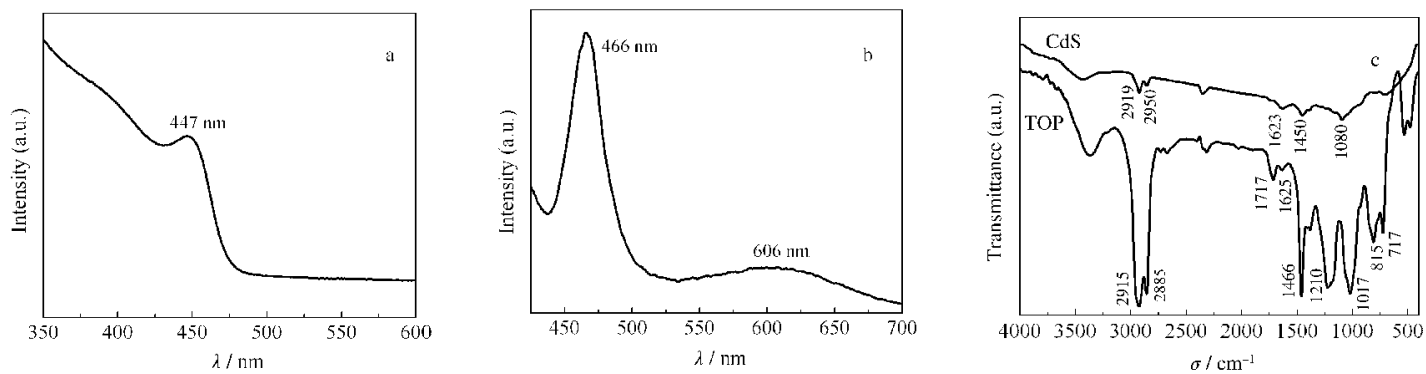

图 $3 \mathrm{CdS}$ 纳米棒的紫外-可见光吸收谱图(a), 荧光发射谱图(b)和傅里叶变换红外光谱图(c)

Fig.3 Optical absorption (a), photoluminescence (b), and FTIR (c) spectra of CdS nanorods preparation condition: $20 \mathrm{mg}$ cadmium acetate in $3 \mathrm{~mL}$ TOP and $12 \mathrm{mg} \mathrm{S}$ powder in $1 \mathrm{~mL}$ TOP reacting at $260{ }^{\circ} \mathrm{C}$ for $4 \mathrm{~h}$ 
处的峰基本一致, 表明反应过程中有三辛基氧化膦 生成, 具体反应方程如反应(4)所示. 结合 $\mathrm{PbSe}$ 纳米 晶 ${ }^{[30]}$ 的形成机理, 提出与此类似的 CdS 纳米晶形成 机理:

$$
\text { }
$$

\section{3 结 论}

利用三辛基膦作为配位溶剂, 控制 $\mathrm{Cd} / \mathrm{S}$ 摩尔比 和浓度制备出具有纤锌矿结构的单分散 CdS 纳米 棒, 该工艺简单高效, 能够用于其他无机纳米材料的 大批量制备. 另外, 在反应过程中只加人三辛基膦作 为单一表面活性剂, 有利于探索 $\mathrm{CdS}$ 纳米晶的形成 和生长机理.

Supporting Information Available free of charge at http://www. whxb.pku.edu.cn.

\section{References}

1 Duan, X.; Huang,Y.; Cui, Y.; Wang, J.; Lieber, C. M. Nature, 2001, 409: 66

2 Kazes, M.; Lewis, D. Y.; Ebenstein, Y.; Mokari, T.; Banin, U. Adv. Mater., 2002, 14: 317

3 Coe, S.; Woo, W. K.; Bawendi, M. G.; Bulovic, V. Nature, 2002, 420: 800

4 Dabbousi, B. O.; Bawendi, M. B.; Onitsuka, O.; Rubner, M. F. Appl. Phys. Lett., 1995, 66: 1316
5 Sykora, M.; Petruska, M. A.; Alstrum-Acevedo, J.; Bezel, I.; Meyer, T. J.; Klimov, V. I. J. Am. Chem. Soc., 2006, 128: 9984

6 Huynh, W. U.; Dittmer, J. J.; Alivisatos, A. P. Science, 2002, 295 : 2425

7 Suri, P.; Mehra, R. M. Solar Energy Materials \& Solar Cells, 2007, 91: 518

8 Gai, H. D.; Wu, Y. S.; Wu, L. L.; Shi, Y. C.; Jing, M.; Zou, K. Acta Phys. -Chim. Sin., 2008, 24: 157 [盖红德, 吴佑实, 吴莉莉, 石元 昌, 井 敏, 邹 科. 物理化学学报, 2008, 24: 157]

9 Klimov, V. I.; Mikhailovsky, A. A.; Xu, A. M.; Hollingsworth, J. A.; Leatherdale, C. A.; Eisler, H. J.; Bawendi, M. G. Science, 2000, 290: 314

10 Artemyev, M. V.; Woggon, U.; Wannemacher, R.; Jaschinski, H.; Langbein, W. Nano Lett., 2001, 1: 309

11 Maskaly, G. R.; Petruska, M. A.; Nanda, J.; Bezel, I. V.; Schaller, R. D.; Htoon, H.; Pietryga, J. M.; Klimov, V. I. Adv. Mater., 2006, 18: 343

12 Michalet, X.; Pinaud, F.; Bentolila, L. A.; Tsay, J. M.; Doose, S.; Li, J. J.; Sundaresan, G.; Wu, A. M. Science, 2005, 307: 538

13 Alivisatos, A. P.; Gu, W.; Larabell, C. Annu. Rev. Biomed. Eng., 2005, 7: 55

14 Zhang, H.; Wang, L. P.; Xiong, H. M.; Hu, L. H.; Yang, B.; Li, W. Adv. Mater., 2003, 15: 1712

15 Yang, J.; Zeng, J.; Yu, S.; Yang, L.; Zhou, G.; Qian, Y. Chem. Mater., 2000, 12: 3259

16 Li, Y.; Wan, J.; Gu, Z. Mater. Sci. Eng. A, 2000, 286: 106

17 Zhan, J.; Yang, X.; Wang, D.; Li, S.; Xie, Y.; Xia, Y.; Qian, Y. Adv. Mater., 2000, 12: 1348

18 Xu, D.; Xu, Y.; Chen, D.; Guo, G.; Gui, L.; Tang, Y. Chem. Phys. Lett., 2000, 325: 340

19 Barrelet, C. J.; Wu, Y.; Bell, D. C.; Lieber, C. M. J. Am. Chem. Soc., 2003, 125: 11498

20 Robison, R. D.; Sadtler, B.; Demchenko, D. O.; Erdonmez, C. K.; Wang, L. W.; Alivisators, A. P. Science, 2007, 317: 355

21 Murray, C. B.; Norris, D. J.; Bawendi, M. G. J. Am. Chem. Soc., 1993, 115: 8706

22 Kang, C. C.; Lai, C. W.; Peng, H. C.; Shyue, J. J.; Chou, P. T. Small, 2007, 3: 1882

23 Peng, X. G.; Manna, L.; Yang, W. D.; Wickham, J.; Scher, E.; Kadavanich, A.; Alivisatos, A. P. Nature, 2000, 404: 59

24 Jun, Y. W.; Lee, S. M.; Kang, N. J.; Cheon, J. J. Am. Chem. Soc., 2001, 123: 5150

25 Prashan, N.; Xu, H.; Peng, X. Nano Lett., 2006, 6: 720

26 Peng, X. G. Adv. Mater., 2003, 15: 459

27 Weller, H. Angew. Chem. Int. Ed. Engl., 1993, 32: 41

28 Cai, W.; Li, Z. G.; Sui, J. H. Nanotechnology, 2008, 19: 465606

29 Butty, J.; Peyghambarian, N.; Kao, Y. H.; Mackenzie, J. D. Appl. Phy. Lett., 1996, 69: 3224

30 Steckel, J. S.; Yen, B. K. H.; Oertel, D. C.; Bawendi, M. G. J. Am. Chem. Soc., 2006, 128: 13032

31 Liu, H. T.; Owen, J. S.; Alivisators, A. P. J. Am. Chem. Soc., 2007, 129: 305 